\title{
Predictors of survival in patients with sarcoma admitted to the intensive care unit
}

Rohan Gupta', Neda Heshami' ${ }^{1}$, Chouhan Jay', Naveen Ramesh², Juhee Song 3 , Xiudong Lei ${ }^{3}$, Erfe Jean Rose ${ }^{4}$, Kristen Carter ${ }^{5}$, Dejka M. Araujo ${ }^{5}$, Robert S. Benjamin ${ }^{5}$, Shreyaskumar Patel ${ }^{5}$, Joseph L. Nates ${ }^{4}$ and Vinod Ravi $i^{*}$

\begin{abstract}
Background: Advances in treatment of sarcoma patients has prolonged survival but has led to increased disease- or treatment-related complications resulting in greater number of admissions to the intensive care unit (ICU). Survival and long-term outcome information about such critically ill patients with sarcoma is unknown.

Methods: The primary objective of the study was to determine the ICU and post-ICU survival rates of critically ill sarcoma patients. Secondary objectives included determining the modifiable and non-modifiable predictors of poor survival. We performed a retrospective chart review of sarcoma patients admitted to the ICU at The University of Texas MD Anderson Cancer Center between January 1, 2005, and December 31, 2012. Main outcome measures were ICU mortality, in-hospital mortality and 1,2, and 6-month survival rates. Covariates such as histological diagnosis, disease characteristics, chemotherapy use, Charlson comorbidity index, Sequential Organ Failure Assessment (SOFA) scores, and clinical findings leading to ICU admission were analyzed for their effects on survival.
\end{abstract}

Results: We identified 172 admissions over the 8-year study period hat met our inclusion criteria. The study population was $45.9 \%$ males with a median age of 52 years. The most common sarcoma subgroups were high-grade unclassified sarcoma (25\%) and bone tumors (17.4\%). The ICU mortality rate was $23.3 \%$ (95\% confidence interval [CI], 16.9-29.6\%), and an additional $6.4 \%$ of patients died before hospital discharge ( $95 \%$ Cl, 22.9-37.1 \%). 6-month OS rates were $41 \%$. The median SOFA scores on admission were 6 (inter quartile range (IQR), 3.5-9) in ICU survivors and 10 (IQR, 6.5-14) in ICU non-survivors. Increase in SOFA scores $\geq 6$ led to poor outcomes (ICU survival $13.3 \%$, OS $6.7 \%$ ). Charlson comorbidity index (HR 1.139, $95 \% \mathrm{Cl} 1.023-1.268, \mathrm{p}=0.02)$ and discharge SOFA scores (HR 1.210, $95 \% \mathrm{Cl}$ $1.141-1.283, \mathrm{p}<0.0001)$ correlated with overall survival.

Conclusions: Our results suggest that patients that are admitted to the ICU for respiratory failure, cardiac arrest, septic shock, acute renal failure or acidosis and also have a high SOFA score with subsequent worsening in the ICU have poor prognosis. Based on the retrospective data which needs further validation we can recommend that judicious approach should be taken in patients with predictors of poor survival before subjecting them to aggressive treatment.

Keywords: Cancer, Sarcoma, ICU, Survival, SOFA

\footnotetext{
*Correspondence: vravi@mdanderson.org

${ }^{5}$ Department of Sarcoma Medical Oncology, The University of Texas MD

Anderson Cancer Center, 1515 Holcombe Blvd \# 450, Houston, TX 77030,

USA

Full list of author information is available at the end of the article
} provided you give appropriate credit to the original author(s) and the source, provide a link to the Creative Commons license, and indicate if changes were made. The Creative Commons Public Domain Dedication waiver (http://creativecommons.org/ publicdomain/zero/1.0/) applies to the data made available in this article, unless otherwise stated. 


\section{Background}

Sarcomas are a rare, histologically and behaviorally diverse group of malignant connective tissue tumors that make up approximately $1 \%$ of all adult malignancies and $12 \%$ of pediatric cancers $[1,2]$. As a result of the substantial progress made in the past 2 decades in understanding the behavior and molecular pathogenesis of sarcoma, new therapies have been developed. Advances in treatment may prolong survival but can lead to increased disease- or treatment-related complications requiring aggressive critical care. Due to the rarity of this class of tumors, the survival of critically ill sarcoma patients has not been well studied.

The treatment of such patients requires a multidisciplinary approach, with coordination among oncologists, critical care physicians, consulting services, ancillary staff, and patients' families. Despite the availability of advanced life support devices in intensive care centers in the United States, it is difficult for both patients and physicians to objectively determine the effect of such heroic measures on prognosis or quality of life. Studies up to the 1990s had shown that among all diseases, patients with cancer had the lowest intensive care unit (ICU) survival rates, and the majority of these patients died soon after their hospital discharge [3-5]. However, over the past two decades, the approach to treatment in such cases has been shifting. Some studies have shown that patients lacking predictors of poor survival outcomes are considered as good candidates for aggressive therapy [6-8].

The primary objective of our study was to determine the ICU and post-ICU survival rates of critically ill sarcoma patients. Secondary objectives included determining the modifiable and nonmodifiable predictors of poor survival.

\section{Methods}

\section{Patient population}

After obtaining institutional review board approval, we retrospectively reviewed the electronic medical records of 212 critically ill patients with sarcoma who had been admitted to the ICU at The University of Texas MD Anderson Cancer Center between January 1, 2006, and December 31, 2012. For the purpose of this analysis, we limited the study population to patient's first ICU admission only. We also excluded from the study population patients who had been admitted to the ICU for perioperative care.

\section{Study design}

This retrospective study was designed to identify predictors of poor survival that contribute to ICU mortality. ICU mortality was defined as the percentage of patients with sarcoma who died in the ICU among total number of patients admitted to the ICU with sarcoma during the study period. Secondary outcomes were in-hospital mortality and 1, 2, and 6-month survival rates, which were defined by similar means. Overall survival (OS) for each patient was measured from the time of initial ICU admission to the last date of contact or death. We defined ICU survival as short-term or acute survival and 6-month survival as long-term survival. Median follow-up time was calculated from the date of ICU admission.

Patient's current and previous chemotherapy regimens were examined to evaluate the impact of various chemotherapy treatments on survival. Tumor burden was noted by recording the various sites of metastasis, such as head and neck, musculoskeletal, heart, lung, liver, gastrointestinal tract, and spleen. The Charlson comorbidities index (CCI) was used to assess the role of serious comorbid disease in the survival of patients in the study [9].

Clinical findings present at the time of ICU admission were recorded to assess acute illness (definitions in parentheses). Variables recorded included acute renal failure, anemia, thrombocytopenia, pancytopenia, hemorrhage (including bleeding from gastrointestinal tract), lactic acidosis, heart failure, pulmonary embolism, respiratory failure (use of noninvasive or invasive mechanical ventilation), cardiac arrest, atrial fibrillation, cardiac dysrhythmia, pneumonia, septic shock, hypotension (systolic blood pressure $<100$ or on vasopressors), hypertension, neutropenic fever (absolute neutrophil count $<1500$ ), altered mental status, and malnutrition (patient on feeding tube or total parental nutrition).

Severity of illness at the time of ICU admission was measured by Sequential Organ Failure Assessment (SOFA) scores. Maximum SOFA scores and discharge SOFA scores were also obtained to track the patients' progress while they were in the ICU. Change in SOFA scores were calculated by subtracting the admission SOFA scores from the maximum SOFA scores. Organ failure at the time of ICU admission was determined by an admission SOFA score $\geq 2$ per organ system. The total number of organ failures was calculated for each patient.

\section{Analysis}

Patient characteristics were tabulated and compared between groups by using the Chi square test or Fisher exact test as appropriate for categorical variables and by the nonparametric Wilcoxon rank sum test for continuous variables. A multivariate logistic regression model was fitted to examine the relationship between death in the ICU and clinical characteristics. Patients who were lost to follow-up or alive were censored at their dates of last contact. The Kaplan-Meier product limit method was used to estimate the survival outcomes of all patients by groups; the log-rank statistic was used to compare groups. 
Cox proportional hazards models were fitted to determine the association of patient and clinical characteristics with OS. Variables that had significant univariate logrank $\mathrm{p}$ values were candidates for the multivariate model. Results were expressed in hazard ratios (HRs), odds ratios (ORs) and $95 \%$ confidence intervals (CIs). p values of less than 0.05 were considered statistically significant; all tests were two-sided. Statistical analyses were carried out by using SAS 9.4 (SAS Institute Inc., Cary, NC) and S-Plus 8.2 (TIBCO Software Inc, Palo Alto, CA, USA).

\section{Results}

\section{Patient characteristics}

We identified a total of 212 sarcoma admissions to the ICU at MD Anderson between January 1, 2005, and December 31, 2012. We excluded 23 ICU admissions of patients who were admitted to the ICU multiple times during the study course. Of the remaining 189 admissions, 17 perioperative admissions were excluded, leaving a sample of 172 first-time ICU admissions. The study population was $45.9 \%$ male with median age of 52 years (interquartile range [IQR] 38-62 years) (Supplemental Digital Content-Table 1). The most common sarcoma subgroups were unclassified high-grade sarcoma (25\%), bone sarcoma (Ewing sarcoma, osteosarcoma, and chondrosarcoma; $17.4 \%$ ), vascular sarcoma (angiosarcoma and epithelioid hemangioendothelioma; $9.9 \%$ ), and leiomyosarcoma (7.6 \%). The ICU mortality rate was $23.3 \%$ (95 \% CI 16.9-29.6\%), and the hospital mortality rate was 29.7 \% (95 \% CI 22.9-37.1 \%). The median Charlson comorbidity index was 6 (IQR 6-7) owing to presence of metastatic cancer in most of the patients at the time of admission.

\section{Death in the ICU}

There were 40 patient deaths $(23.3 \%)$ in the ICU. In the univariate logistic regression model for the death at the ICU, short-term mortality did not correlate with tumor site, histology, disease status, presence of metastatic disease, Charlson comorbidity index or neutropenia at the time of ICU admission. However, patients with clinical findings of acidosis, acute renal failure, cardiac arrest, hypotension (including septic shock), pneumonia, septic shock or respiratory failure at the time of ICU admission had worse outcomes than patients who lacked these findings on admission (Supplemental Digital ContentTables 1, 2).

The median SOFA scores on admission were 6 (IQR 3.5-9) in ICU survivors and 10 (IQR 6.5-14) in non-survivors. In addition, the median maximum SOFA scores were 7 (IQR 4-9.5) in survivors and 14 (IQR 10-17) in non-survivors, and the median discharge SOFA scores were 4 (IQR 2-6) in survivors and 10 (IQR, 7.5-13.5) in non-survivors. Patients with admission SOFA scores of 11 or more had a lower ICU survival rate than did those with scores less than 11 (45.7 vs $80 \%$ or more) (Table 2). An increase of 6 or more in the SOFA score from the time of admission significantly affected short- (ICU survival $13.3 \%$ ) and long-term outcomes (OS $6.7 \%$ ) (Table 3).

Multivariate logistic regression model for death in the ICU showed that SOFA admission score (OR 1.23, $95 \%$ CI 1.12-1.35, p < 0.0001) was associated with death in the ICU. Variables that were initially included in the model and then reduced in a stepwise selection were SOFA admission score and number of metastatic sites.

\section{OS from ICU admission}

Median follow-up among all patients was 2.9 months (range 0.02-77.5 months). At the time of this analysis, 135 patients $(78.5 \%)$ had died. For the whole cohort, 1, 2, and 6-month OS rates were 64,57, and $41 \%$, respectively (Table 4). The Kaplan-Meier curves for OS are shown in Fig. 1.

In multivariable Cox proportional hazards models (Table 5), patients with gastrointestinal stromal tumors (HR 0.281, $95 \%$ CI 0.119-0.662, p = 0.004) and leiomyosarcoma (HR 0.375, $95 \%$ CI 0.160-0.880, $\mathrm{p}=0.02$ ) had a lower risk of death than did patients with unclassified high-grade sarcoma. Patients with higher Charlson Comorbidity Index (HR 1.139, 95 \% CI 1.023-1.268, $\mathrm{p}=0.02)$ and those with higher SOFA scores at discharge (HR 1.210, 95 \% CI 1.141-1.283, p < 0.0001) also had higher risk of death (Table 5). Median survival rates were lower in patients with acute renal failure, cardiac arrest, pneumonia, septic shock, and respiratory failure.

\section{Discussion}

Decisions about the intensive care treatment of critically ill cancer patients with poor prognoses are challenging and need to be evaluated on a patient-by-patient basis. Our study benefits both physicians and family members by providing objective data on ICU mortality, long-term survival, and objective predictors of survival. Our results showed that among sarcoma patients, the ICU mortality, in-hospital mortality, and long-term survival rates were $23.3,29.7$, and $41 \%$, respectively. Patients who were admitted due to acute renal failure, cardiac arrest, septic shock, or respiratory failure had poor ICU outcomes and median survival durations ranging from 1 to 21 days. The median SOFA scores at the times of admission and discharge were significantly lower in ICU survivors than in non-survivors. An increase in SOFA score during the ICU stay is an important predictor of poor survival outcomes. We also determined that a higher number of organ failures was associated with an increased risk of ICU mortality (Supplemental Digital Content-Table 1). 
Table 1 Patient and clinical characteristics by Alive or Death at the Intensive Care Unit (ICU)

\begin{tabular}{|c|c|c|c|c|}
\hline & $\begin{array}{l}\text { All patients }{ }^{a} \\
(N=172)\end{array}$ & $\begin{array}{l}\text { Alive at ICU discharge } \\
(\mathrm{N}=132)\end{array}$ & $\begin{array}{l}\text { Death at ICU } \\
(N=40)\end{array}$ & $\mathbf{p}^{*}$ \\
\hline \multicolumn{5}{|l|}{ Age (y) } \\
\hline Median (IQR) & $52(38-62)$ & $52(38-62)$ & $53.5(36-66)$ & 0.65 \\
\hline \multicolumn{5}{|l|}{ Gender } \\
\hline Female & $93(54.1 \%)$ & $71(76.3 \%)$ & $22(23.7 \%)$ & \\
\hline Male & $79(45.9 \%)$ & $61(77.2 \%)$ & $18(22.8 \%)$ & $0.89^{\dagger}$ \\
\hline \multicolumn{5}{|l|}{ Histological diagnosis } \\
\hline Unclassified high-grade sarcoma & $43(25.0 \%)$ & $36(83.7 \%)$ & $7(16.3 \%)$ & \\
\hline Bone sarcomac & $30(17.4 \%)$ & $21(70 \%)$ & $9(30 \%)$ & \\
\hline Vascular $^{d}$ & $17(9.9 \%)$ & $16(94.1 \%)$ & $1(5.9 \%)$ & \\
\hline $\mathrm{GIST}^{\mathrm{e}}$ & $11(6.4 \%)$ & $10(90.9 \%)$ & $1(9.1 \%)$ & \\
\hline $\mathrm{MFH}^{\mathrm{e}}$ & $11(6.4 \%)$ & $6(54.5 \%)$ & $5(45.5 \%)$ & \\
\hline Muscle & $10(5.8 \%)$ & $7(70 \%)$ & $3(30 \%)$ & \\
\hline Leiomyosarcoma & $13(7.6 \%)$ & $12(92.3 \%)$ & $1(7.7 \%)$ & \\
\hline Liposarcoma & $7(4.1 \%)$ & 4 (57.1\%) & $3(42.9 \%)$ & \\
\hline Synovial sarcoma & $9(5.2 \%)$ & $5(55.6 \%)$ & $4(44.4 \%)$ & \\
\hline Others & $21(12.2 \%)$ & $15(71.4 \%)$ & $6(28.6 \%)$ & 0.09 \\
\hline \multicolumn{5}{|l|}{ Status of malignancy } \\
\hline First course of chemotherapy & $29(16.9 \%)$ & $20(69 \%)$ & $9(31 \%)$ & \\
\hline Progression & $59(34.3 \%)$ & $45(76.3 \%)$ & $14(23.7 \%)$ & \\
\hline Stable disease or partial remission & $61(35.5 \%)$ & $50(82 \%)$ & $11(18 \%)$ & \\
\hline Complete remission & $4(2.3 \%)$ & $4(100 \%)$ & $0(0 \%)$ & \\
\hline Mixed response & $6(3.5 \%)$ & $4(66.7 \%)$ & $2(33.3 \%)$ & \\
\hline Unknown & $13(7.6 \%)$ & $9(69.2 \%)$ & $4(30.8 \%)$ & 0.57 \\
\hline \multicolumn{5}{|l|}{ Site of malignancy } \\
\hline Head and neck & $15(8.7 \%)$ & $11(73.3 \%)$ & $4(26.7 \%)$ & \\
\hline Thoracic & $43(25 \%)$ & $34(79.1 \%)$ & $9(20.9 \%)$ & \\
\hline Abdomen & $71(41.3 \%)$ & $55(77.5 \%)$ & $16(22.5 \%)$ & \\
\hline Extremities & $43(25 \%)$ & $32(74.4 \%)$ & $11(25.6 \%)$ & 0.94 \\
\hline \multicolumn{5}{|l|}{ Organ metastasis } \\
\hline \multicolumn{5}{|l|}{ Lung } \\
\hline No & $86(50 \%)$ & $69(80.2 \%)$ & $17(19.8 \%)$ & \\
\hline Yes & $86(50 \%)$ & $63(73.3 \%)$ & $23(26.7 \%)$ & 0.28 \\
\hline \multicolumn{5}{|l|}{ Liver } \\
\hline No & $142(82.6 \%)$ & $108(76.1 \%)$ & $34(23.9 \%)$ & \\
\hline Yes & $30(17.4 \%)$ & $24(80 \%)$ & $6(20 \%)$ & 0.64 \\
\hline \multicolumn{5}{|l|}{ Other } \\
\hline No & 79 (45.9\%) & $57(72.2 \%)$ & $22(27.8 \%)$ & \\
\hline Yes & $93(54.1 \%)$ & $75(80.6 \%)$ & $18(19.4 \%)$ & 0.19 \\
\hline \multicolumn{5}{|l|}{ Number of organ metastasis } \\
\hline 0 & $42(24.4 \%)$ & $32(76.2 \%)$ & $10(23.8 \%)$ & \\
\hline 1 & $54(31.4 \%)$ & $39(72.2 \%)$ & $15(27.8 \%)$ & \\
\hline$\geq 2$ & $76(44.2 \%)$ & $61(80.3 \%)$ & $15(19.7 \%)$ & 0.56 \\
\hline \multicolumn{5}{|l|}{ Treatment } \\
\hline \multicolumn{5}{|l|}{ Current chemotherapy regimen } \\
\hline None & $43(25 \%)$ & $34(79.1 \%)$ & $9(20.9 \%)$ & \\
\hline Adriamycin-based & $68(39.5 \%)$ & $50(73.5 \%)$ & $18(26.5 \%)$ & \\
\hline Gemcitabine-based & $20(11.6 \%)$ & $13(65 \%)$ & $7(35 \%)$ & \\
\hline Targeted therapy & $41(23.8 \%)$ & $35(85.4 \%)$ & $6(14.6 \%)$ & 0.29 \\
\hline
\end{tabular}


Table 1 continued

\begin{tabular}{|c|c|c|c|c|}
\hline & $\begin{array}{l}\text { All patients } \\
(\mathrm{N}=172)\end{array}$ & $\begin{array}{l}\text { Alive at ICU discharge }{ }^{b} \\
(N=132)\end{array}$ & $\begin{array}{l}\text { Death at ICU } \\
(N=40)\end{array}$ & $\mathbf{p}^{*}$ \\
\hline $\begin{array}{l}\text { No. cycles of current chemotherapy, median } \\
\left(\text { IQR }^{\mathrm{e}}\right)\end{array}$ & $1(0-3)$ & $1(0-3)$ & $1(1-2)$ & $0.80^{\dagger}$ \\
\hline No. cycles of prior chemotherapies, median (IQR) & $1(0-3)$ & $1(0-3)$ & $0(0-2)$ & $0.037^{\dagger}$ \\
\hline \multicolumn{5}{|l|}{ No. cycles of prior chemotherapies } \\
\hline $0-1$ & $114(66.3 \%)$ & $88(77.2 \%)$ & $26(22.8 \%)$ & \\
\hline$\geq 2$ & $58(33.7 \%)$ & $44(75.9 \%)$ & $14(24.1 \%)$ & 0.85 \\
\hline \multicolumn{5}{|l|}{ Radiation } \\
\hline No & $109(63.7 \%)$ & $80(73.4 \%)$ & $29(26.6 \%)$ & \\
\hline Yes & $62(36.3 \%)$ & $51(82.3 \%)$ & $11(17.7 \%)$ & 0.19 \\
\hline \multicolumn{5}{|l|}{ Clinical conditions present at ICU admission } \\
\hline Anemia & $144(83.7 \%)$ & $110(76.4 \%)$ & $34(23.6 \%)$ & 0.80 \\
\hline Hypotension & $92(53.5 \%)$ & $63(68.5 \%)$ & $29(31.5 \%)$ & 0.006 \\
\hline Septic shock & $53(30.8 \%)$ & $33(62.3 \%)$ & $20(37.7 \%)$ & 0.003 \\
\hline Bacteremia & $21(12.2 \%)$ & $14(66.7 \%)$ & 7 (33.3\%) & 0.24 \\
\hline Thrombocytopenia & $86(5 \%)$ & 68 (79.1\%) & 18 (20.9\%) & 0.47 \\
\hline Respiratory failure & $74(43 \%)$ & $39(52.7 \%)$ & $35(47.3 \%)$ & $<0.0001$ \\
\hline Acidosis & $70(40.7 \%)$ & $42(60 \%)$ & $28(40 \%)$ & $<0.0001$ \\
\hline Altered mental status & $65(37 \%)$ & $42(64.6 \%)$ & $23(35.4 \%)$ & 0.003 \\
\hline Abnormal glucose & $60(34.9 \%)$ & $42(70 \%)$ & $18(30 \%)$ & 0.13 \\
\hline Acute renal failure & $58(33.7 \%)$ & $35(60.3 \%)$ & $23(39.7 \%)$ & 0.0003 \\
\hline Pancytopenia & $58(33.7 \%)$ & $47(81 \%)$ & $11(19 \%)$ & 0.34 \\
\hline Pneumonia & $51(29.7 \%)$ & $32(62.7 \%)$ & $19(37.3 \%)$ & 0.005 \\
\hline Neutropenia $\left(\right.$ ANC $^{\mathrm{e}}<1500 / \mathrm{mm}^{3}$ ) & $51(29.7 \%)$ & $40(78.4 \%)$ & $11(21.6 \%)$ & 0.73 \\
\hline Cardiac dysrhythmia & 35 (20.3\%) & $23(65.7 \%)$ & $12(34.3 \%)$ & 0.08 \\
\hline Heart failure & $32(18.6 \%)$ & $21(65.6 \%)$ & $11(34.4 \%)$ & 0.10 \\
\hline Hypertension & $25(14.5 \%)$ & $22(88 \%)$ & $3(12 \%)$ & $0.20^{*}$ \\
\hline Malnutrition (protein/calorie) NOS & $23(13.4 \%)$ & $15(65.2 \%)$ & $8(34.8 \%)$ & 0.16 \\
\hline Hemorrhage & $15(8.7 \%)$ & $12(80 \%)$ & $3(20 \%)$ & $1.0^{*}$ \\
\hline Gastrointestinal hemorrhage & $13(7.6 \%)$ & $12(92.3 \%)$ & $1(7.7 \%)$ & $0.30^{*}$ \\
\hline Cardiac arrest & $11(6.4 \%)$ & $3(27.3 \%)$ & $8(72.7 \%)$ & $0.0004^{*}$ \\
\hline Pulmonary embolism & $11(6.4 \%)$ & $8(72.7 \%)$ & $3(27.3 \%)$ & $0.72^{*}$ \\
\hline Atrial fibrillation & $10(5.8 \%)$ & $7(70 \%)$ & $3(30 \%)$ & $0.70^{*}$ \\
\hline Seizures/convulsions & $9(5.2 \%)$ & $8(88.9 \%)$ & $1(11.1 \%)$ & $0.69^{*}$ \\
\hline \multicolumn{5}{|l|}{ ICU admission data } \\
\hline Mechanical v entilator & $75(43.6 \%)$ & $40(30.3 \%)$ & $35(87.5 \%)$ & $<0.0001$ \\
\hline Charlson comorbidity index, median (IQR) & $6(6-7)$ & $6(6-7)$ & $6(4.5-7)$ & $0.70^{\dagger}$ \\
\hline$\leq 2$ & $26(15.1 \%)$ & $19(73.1 \%)$ & $7(26.9 \%)$ & \\
\hline$>2$ & $146(84.9 \%)$ & $113(77.4 \%)$ & $33(22.6 \%)$ & 0.63 \\
\hline SOFA admission score, median (IQR) & $7(4-10)$ & $6(3.5-9)$ & $10(6.5-14)$ & $<0.0001^{\dagger}$ \\
\hline Max SOFA admission score, median (IQR) & $8(5-12)$ & $7(4-9.5)$ & $14(10-17)$ & $<0.0001^{\dagger}$ \\
\hline SOFA discharge score, median (IQR) & $5(3-8)$ & $4(2-6)$ & $10(7.5-13.5)$ & $<0.0001^{\dagger}$ \\
\hline No. organ failures, median (IQR) & $1(0-2)$ & $1(0-2)$ & $2(1-3)$ & $<0.0001$ \\
\hline
\end{tabular}

* Fisher exact $p$ value

+ Wilcoxon rank-sum test

a Count (column \%-percent of admissions with that variable) are presented unless specified

${ }^{b}$ Count (row \%-percent of $\mathrm{N}$ in column with All patients) are presented unless specified

c Ewing sarcoma, osteosarcoma, chondrosarcoma

d Angiosarcoma, epithelioid hemangioendothelioma

e Gastrointestinal stromal tumor, Malignant fibrous histiocytoma, Interquartile range, Sequential Organ Failure Assessment, Absolute Neutrophil Count 
Table 2 Univariate logistic regression model for death at ICU

\begin{tabular}{|c|c|c|c|c|}
\hline & Odds Ratio & $95 \% \mathrm{C}$ & & $\mathrm{p}$ \\
\hline Reason for ICU admission acidosis: yes v. no & 5.000 & 2.317 & 10.788 & $<0.0001$ \\
\hline Reason for ICU admission renal failure: yes v. no & 3.750 & 1.795 & 7.831 & 0.0004 \\
\hline Reason for ICU admission cardiac arrest: yes v. no & 10.750 & 2.699 & 42.824 & 0.0008 \\
\hline Reason for ICU admission cardiac dysrhythmia: yes v. no & 2.031 & 0.902 & 4.575 & 0.0873 \\
\hline Reason for ICU admission hypotension: yes v. no & 2.887 & 1.332 & 6.258 & 0.0072 \\
\hline Reason for ICU admission pneumonia: yes v. no & 2.827 & 1.353 & 5.910 & 0.0057 \\
\hline Reason for ICU admission respiratory failure: yes v. no & 16.691 & 6.086 & 45.775 & $<0.0001$ \\
\hline Reason for ICU admission septic shock: yes v. no & 3.000 & 1.439 & 6.253 & 0.0034 \\
\hline Number of organ failures & 1.933 & 1.417 & 2.637 & $<0.0001$ \\
\hline Number of mets & 0.997 & 0.745 & 1.334 & 0.9835 \\
\hline \multicolumn{5}{|l|}{ Histology diagnosis } \\
\hline Unclassified high grade sarcoma & 1.000 & & & \\
\hline Bone (Ewing, Osteo, Chondrosarcoma) & 2.204 & 0.716 & 6.788 & 0.1685 \\
\hline Vascular & 0.321 & 0.036 & 2.833 & 0.3068 \\
\hline GIST & 0.514 & 0.056 & 4.685 & 0.5552 \\
\hline $\mathrm{MFH}$ & 4.286 & 1.019 & 18.029 & 0.0471 \\
\hline Muscle & 2.204 & 0.456 & 10.661 & 0.3258 \\
\hline Leiomyosarcoma & 0.429 & 0.048 & 3.848 & 0.4493 \\
\hline Liposarcoma & 3.857 & 0.703 & 21.153 & 0.1200 \\
\hline Synovial sarcoma & 4.114 & 0.878 & 19.270 & 0.0726 \\
\hline Others & 2.057 & 0.592 & 7.149 & 0.2564 \\
\hline
\end{tabular}

Table 3 Trends in Sequential Organ Failure Assessment (SOFA) scores and their impact on ICU and overall survival

\begin{tabular}{|c|c|c|c|c|}
\hline $\begin{array}{l}\text { Increase in SOFA score during ICU } \\
\text { stay (Maximum SOFA- Admission } \\
\text { SOFA) }\end{array}$ & $\begin{array}{l}\text { No. patients in } \\
\text { subgroup }\end{array}$ & $\begin{array}{l}\text { Patients surviving ICU } \\
\text { stay, \% }\end{array}$ & $\begin{array}{l}\text { Patients surviving till end } \\
\text { of study, } \%\end{array}$ & $\begin{array}{l}\text { Median survival, } \\
\text { d (range) }\end{array}$ \\
\hline \multicolumn{5}{|l|}{ SOFA scores during ICU stay } \\
\hline 0 or less & 103 & 86.4 & 22.3 & $187(108-347)$ \\
\hline $1-3$ & 43 & 81.4 & 27.9 & $96(21-193)$ \\
\hline $4-5$ & 10 & 60.0 & 10.0 & $12(2-85)$ \\
\hline$\geq 6$ & 15 & 13.3 & 6.7 & $17(6-22)$ \\
\hline \multicolumn{5}{|l|}{ Admission SOFA scores } \\
\hline $0-4$ & 50 & 88.0 & 26.0 & $159(49-289)$ \\
\hline $5-7$ & 46 & 84.8 & 21.7 & $182(38-347)$ \\
\hline $8-10$ & 41 & 80.5 & 24.4 & $185(58-494)$ \\
\hline$\geq 11$ & 35 & 45.7 & 11.4 & $6(2-19)$ \\
\hline \multicolumn{5}{|c|}{ Change in SOFA scores of 35 patients with admission SOFA scores of 11 or more } \\
\hline$\leq 0$ & 18 & 44.4 & 5.6 & $4.5(0.5-9)$ \\
\hline 1 & 6 & 83.3 & 33.3 & $56(4-N E)$ \\
\hline 2 & 3 & 66.7 & 33.3 & $13(2-N E)$ \\
\hline$\geq 3$ & 8 & 12.5 & 0.0 & $6(1-46)$ \\
\hline
\end{tabular}

ADM SOFA SOFA score at the time of ICU admission, MAXOFA maximum SOFA score, NE not estimated

The ICU mortality rates observed in the study population were consistent with those in the existing medical literature and promote a case for a higher level of care with aggressive monitoring in patients who lack predictors of poor outcomes. Our data have shown that adequate cardiac, renal, and respiratory functions play a key role in acute survival. Serial SOFA scores may serve as an objective measure for short-term and long-term prognosis for 
Table 4 Overall survival estimates by patients and clinical characteristics in percentages

\begin{tabular}{|c|c|c|c|c|c|c|}
\hline & No of patients & No of deaths & $\begin{array}{l}\text { 1-month overall } \\
\text { survival estimate } \\
(95 \% \mathrm{Cl})\end{array}$ & $\begin{array}{l}\text { 2-month overall } \\
\text { survival estimate } \\
(95 \% \mathrm{Cl})\end{array}$ & $\begin{array}{l}\text { 6-month overall } \\
\text { survival estimate } \\
(95 \% \mathrm{Cl})\end{array}$ & p \\
\hline All patients & 172 & 135 & $64(57-71)$ & $57(49-64)$ & $41(33-48)$ & \\
\hline \multicolumn{7}{|l|}{ Age (years) } \\
\hline$<65$ & 139 & 109 & $64(56-72)$ & $56(47-64)$ & $41(33-50)$ & \\
\hline$\geq 65$ & 33 & 26 & $64(45-77)$ & $61(42-75)$ & $37(21-54)$ & 0.91 \\
\hline \multicolumn{7}{|l|}{ Gender } \\
\hline Female & 93 & 72 & $66(56-75)$ & $60(49-69)$ & $45(34-55)$ & \\
\hline Male & 79 & 63 & $62(50-71)$ & $48(37-59)$ & $36(25-47)$ & 0.33 \\
\hline \multicolumn{7}{|l|}{ Histological diagnosis } \\
\hline $\begin{array}{l}\text { Unclassified high-grade } \\
\text { sarcoma }\end{array}$ & 43 & 34 & $67(51-79)$ & $58(42-71)$ & $46(30-60)$ & \\
\hline Bones $^{\mathrm{a}}$ & 30 & 25 & $70(50-83)$ & $60(40-75)$ & $38(21-55)$ & \\
\hline Vascular ${ }^{\mathrm{b}}$ & 17 & 16 & $64(36-82)$ & $51(25-72)$ & $32(12-54)$ & \\
\hline GIST & 11 & 7 & $73(37-90)$ & $64(30-85)$ & $45(17-71)$ & \\
\hline MFH & 11 & 8 & $55(23-78)$ & $55(23-78)$ & $36(11-63)$ & \\
\hline Muscle & 10 & 9 & $60(25-83)$ & $60(25-83)$ & $20(3-47)$ & \\
\hline Leiomyosarcoma & 13 & 7 & $84(50-96)$ & $84(50-96)$ & $75(40-91)$ & \\
\hline Liposarcoma & 7 & 7 & $29(4-61)$ & $29(4-61)$ & $29(4-61)$ & \\
\hline Synovial sarcoma & 9 & 6 & $56(20-80)$ & $56(20-80)$ & $56(20-80)$ & \\
\hline Others & 21 & 16 & $56(33-74)$ & $45(23-65)$ & $28(11-49)$ & 0.07 \\
\hline \multicolumn{7}{|l|}{ Status of malignancy } \\
\hline $\begin{array}{l}\text { First course of chemo- } \\
\text { therapy }\end{array}$ & 29 & 22 & $66(45-8)$ & $55(35-71)$ & $41(22-59)$ & \\
\hline Progression & 59 & 56 & $49(36-61)$ & $37(25-49)$ & $17(9-27)$ & \\
\hline $\begin{array}{l}\text { Stable disease or partial } \\
\text { remission }\end{array}$ & 61 & 41 & $75(62-84)$ & $72(58-81)$ & $61(47-72)$ & \\
\hline Complete remission & 4 & 2 & 100 & 100 & $75(13-96)$ & \\
\hline Mixed response & 6 & 5 & $67(19-90)$ & $67(19-90)$ & $67(19-90)$ & \\
\hline Unknown & 13 & 9 & $68(36-87)$ & $68(36-87)$ & $29(7-56)$ & $<0.0001$ \\
\hline \multicolumn{7}{|l|}{ Site of malignancy } \\
\hline Head and neck & 15 & 11 & $52(25-73)$ & $52(25-73)$ & $29(9-53)$ & \\
\hline Thoracic & 43 & 33 & $67(51-79)$ & $60(43-73)$ & $46(30-60)$ & \\
\hline Abdomen & 71 & 57 & $62(49-72)$ & $53(41-64)$ & $37(26-48)$ & \\
\hline Extremities & 43 & 34 & $7(54-81)$ & $63(47-75)$ & $46(31-60)$ & 0.86 \\
\hline \multicolumn{7}{|l|}{ Organ metastasis } \\
\hline \multicolumn{7}{|l|}{ Lung } \\
\hline No & 86 & 62 & $66(55-75)$ & $59(48-69)$ & $46(35-56)$ & \\
\hline Yes & 86 & 73 & $62(51-72)$ & $55(43-65)$ & $36(25-46)$ & 0.02 \\
\hline \multicolumn{7}{|l|}{ Liver } \\
\hline No & 142 & 109 & $66(58-73)$ & $59(51-67)$ & $46(37-54)$ & \\
\hline Yes & 30 & 26 & $56(36-72)$ & $45(27-62)$ & $17(6-33)$ & 0.0093 \\
\hline \multicolumn{7}{|l|}{ Other } \\
\hline No & 79 & 55 & $66(54-75)$ & $63(51-73)$ & $49(38-60)$ & \\
\hline Yes & 93 & 80 & $63(52-72)$ & $52(41-62)$ & $33(24-43)$ & 0.0091 \\
\hline \multicolumn{7}{|c|}{ Number of organ metastases } \\
\hline 0 & 42 & 25 & $71(55-83)$ & $69(53-81)$ & $59(43-72)$ & \\
\hline 1 & 54 & 43 & $59(45-71)$ & $53(39-65)$ & $40(26-53)$ & \\
\hline $2+$ & 76 & 67 & $64(52-74)$ & $53(41-64)$ & $31(21-42)$ & 0.001 \\
\hline
\end{tabular}


Table 4 continued

\begin{tabular}{|c|c|c|c|c|c|c|}
\hline & No of patients & No of deaths & $\begin{array}{l}\text { 1-month overall } \\
\text { survival estimate } \\
(95 \% \mathrm{Cl})\end{array}$ & $\begin{array}{l}\text { 2-month overall } \\
\text { survival estimate } \\
(95 \% \mathrm{Cl})\end{array}$ & $\begin{array}{l}\text { 6-month overall } \\
\text { survival estimate } \\
(95 \% \mathrm{Cl})\end{array}$ & p \\
\hline \multicolumn{7}{|l|}{ Localized disease } \\
\hline No & 130 & 110 & $62(53-7)$ & $53(44-61)$ & $34(26-43)$ & \\
\hline Yes & 42 & 25 & $71(55-83)$ & $69(53-81)$ & $59(43-72)$ & $<0.0001$ \\
\hline \multicolumn{7}{|l|}{ Treatment } \\
\hline \multicolumn{7}{|c|}{ Current chemotherapy regimen } \\
\hline None & 43 & 31 & $57(41-7)$ & $52(36-66)$ & $39(24-53)$ & \\
\hline $\begin{array}{l}\text { Adriamycin-based } \\
\text { chemotherapy }\end{array}$ & 68 & 51 & $69(56-78)$ & $66(53-76)$ & $51(38-63)$ & \\
\hline $\begin{array}{l}\text { Gemcitabine-based } \\
\text { therapy }\end{array}$ & 20 & 18 & $5(27-69)$ & $45(23-65)$ & $25(9-45)$ & \\
\hline Targeted therapy & 41 & 35 & $71(54-82)$ & $54(37-67)$ & $34(20-49)$ & 0.31 \\
\hline \multicolumn{7}{|l|}{ Radiation } \\
\hline No & 109 & 85 & $63(54-72)$ & $59(49-67)$ & $39(29-48)$ & \\
\hline Yes & 62 & 50 & $65(52-76)$ & $53(40-65)$ & $43(30-55)$ & 0.86 \\
\hline \multicolumn{7}{|c|}{ Clinical conditions present at ICU admission } \\
\hline Anemia & 144 & 113 & $66(57-73)$ & $59(50-66)$ & $43(35-51)$ & 0.60 \\
\hline Hypotension & 92 & 73 & $57(46-66)$ & $51(40-61)$ & $42(32-52)$ & 0.96 \\
\hline Septic shock & 53 & 44 & $49(35-62)$ & $45(32-58)$ & $33(21-46)$ & 0.10 \\
\hline Bacteremia & 21 & 17 & $57(34-75)$ & $57(34-75)$ & $43(22-62)$ & 0.58 \\
\hline Thrombocytopenia & 86 & 63 & $71(60-79)$ & $64(52-73)$ & $49(37-59)$ & 0.037 \\
\hline Respiratory failure & 74 & 62 & $46(34-56)$ & $41(30-52)$ & $26(16-37)$ & $<0.0001$ \\
\hline Acidosis & 70 & 58 & $47(35-58)$ & $41(30-53)$ & $35(24-46)$ & 0.011 \\
\hline Altered mental status & 65 & 57 & $52(39-63)$ & $42(30-54)$ & $25(15-36)$ & 0.001 \\
\hline Abnormal glucose & 60 & 50 & $60(46-71)$ & $49(36-61)$ & $25(14-37)$ & 0.017 \\
\hline Acute renal failure & 58 & 49 & $48(35-60)$ & $40(27-52)$ & $29(18-41)$ & 0.016 \\
\hline Pancytopenia & 58 & 41 & $79(66-88)$ & $74(61-83)$ & $57(43-69)$ & 0.006 \\
\hline Respiratory abnormality & 53 & 38 & $73(59-83)$ & $64(49-75)$ & $49(35-62)$ & 0.036 \\
\hline Pneumonia & 51 & 42 & $53(38-65)$ & $49(35-62)$ & $35(22-48)$ & 0.41 \\
\hline $\begin{array}{l}\text { Neutropenia } \\
\qquad\left(\text { ANC }<1500 / \mathrm{mm}^{3}\right)\end{array}$ & 51 & 36 & $78(64-87)$ & $70(56-81)$ & $58(43-70)$ & 0.013 \\
\hline Cardiac dysrhythmia & 35 & 26 & $57(39-72)$ & $51(34-66)$ & $46(29-61)$ & 0.96 \\
\hline Heart failure & 32 & 25 & $63(44-77)$ & $56(37-71)$ & $53(34-68)$ & 0.80 \\
\hline Hypertension & 25 & 15 & $80(58-91)$ & $71(48-85)$ & $61(39-78)$ & 0.049 \\
\hline $\begin{array}{l}\text { Malnutrition (protein/ } \\
\text { calorie) NOS }\end{array}$ & 23 & 21 & $60(37-77)$ & $60(37-77)$ & $28(11-47)$ & 0.047 \\
\hline $\begin{array}{l}\text { Hemorrhage (non-gas- } \\
\text { trointestinal) }\end{array}$ & 15 & 9 & $64(34-83)$ & $64(34-83)$ & $47(19-71)$ & 0.44 \\
\hline $\begin{array}{l}\text { Other pulmonary insuf- } \\
\text { ficiency }\end{array}$ & 14 & 11 & $71(41-88)$ & $64(34-83)$ & $64(34-83)$ & 0.71 \\
\hline $\begin{array}{l}\text { Gastrointestinal hemor- } \\
\text { rhage }\end{array}$ & 13 & 9 & $62(31-82)$ & $54(25-76)$ & $38(14-63)$ & 0.51 \\
\hline Cardiac arrest & 11 & 8 & $27(7-54)$ & $27(7-54)$ & $27(7-54)$ & 0.17 \\
\hline Pulmonary embolism & 11 & 10 & $73(37-90)$ & $63(29-84)$ & $32(8-59)$ & 0.21 \\
\hline Atrial fibrillation & 10 & 7 & $70(33-89)$ & $70(33-89)$ & $6(25-83)$ & 0.40 \\
\hline Seizures & 9 & 8 & $67(28-88)$ & $44(14-72)$ & $30(5-61)$ & 0.54 \\
\hline \multicolumn{7}{|l|}{ ICU admission data } \\
\hline \multicolumn{7}{|c|}{ Charlson comorbidity index } \\
\hline$\leq 2$ & 26 & 15 & $69(48-83)$ & $69(48-83)$ & $54(33-71)$ & \\
\hline$>2$ & 146 & 120 & $63(55-71)$ & $55(46-62)$ & $38(30-46)$ & 0.0031 \\
\hline
\end{tabular}


Table 4 continued

\begin{tabular}{|c|c|c|c|c|c|c|}
\hline & No of patients & No of deaths & $\begin{array}{l}\text { 1-month overall } \\
\text { survival estimate } \\
(95 \% \mathrm{Cl})\end{array}$ & $\begin{array}{l}\text { 2-month overall } \\
\text { survival estimate } \\
(95 \% \mathrm{Cl})\end{array}$ & $\begin{array}{l}\text { 6-month overall } \\
\text { survival estimate } \\
(95 \% \mathrm{Cl})\end{array}$ & $p$ \\
\hline \multicolumn{7}{|c|}{ Charlson comorbidity index } \\
\hline$<6$ & 42 & 25 & $71(55-83)$ & $69(53-81)$ & $59(43-72)$ & \\
\hline$\geq 6$ & 130 & 110 & $62(53-70)$ & $53(44-61)$ & $34(26-43)$ & $<0.0001$ \\
\hline \multicolumn{7}{|c|}{ SOFA maximum score } \\
\hline$<8$ & 79 & 60 & $82(72-89)$ & $71(60-80)$ & $49(38-60)$ & \\
\hline$\geq 8$ & 93 & 75 & $49(39-59)$ & $45(34-55)$ & $33(24-43)$ & 0.005 \\
\hline \multicolumn{7}{|c|}{ SOFA discharge score } \\
\hline$<5$ & 77 & 59 & $80(69-88)$ & $71(59-80)$ & $47(35-58)$ & \\
\hline$\geq 5$ & 95 & 76 & $51(41-61)$ & $46(36-56)$ & $36(26-45)$ & 0.12 \\
\hline \multicolumn{7}{|c|}{ No. organ failures } \\
\hline 1 & 59 & 47 & $76(63-85)$ & $69(56-79)$ & $53(40-65)$ & \\
\hline$\geq 2$ & 113 & 88 & $58(48-66)$ & $50(41-59)$ & $34(25-43)$ & 0.12 \\
\hline
\end{tabular}

ADM SOFA SOFA score at the time of ICU admission, MAXOFA maximum SOFA score, NE not estimated

a Ewing sarcoma, osteosarcoma, chondrosarcoma

b Angiosarcoma, epithelioid hemangioendothelioma

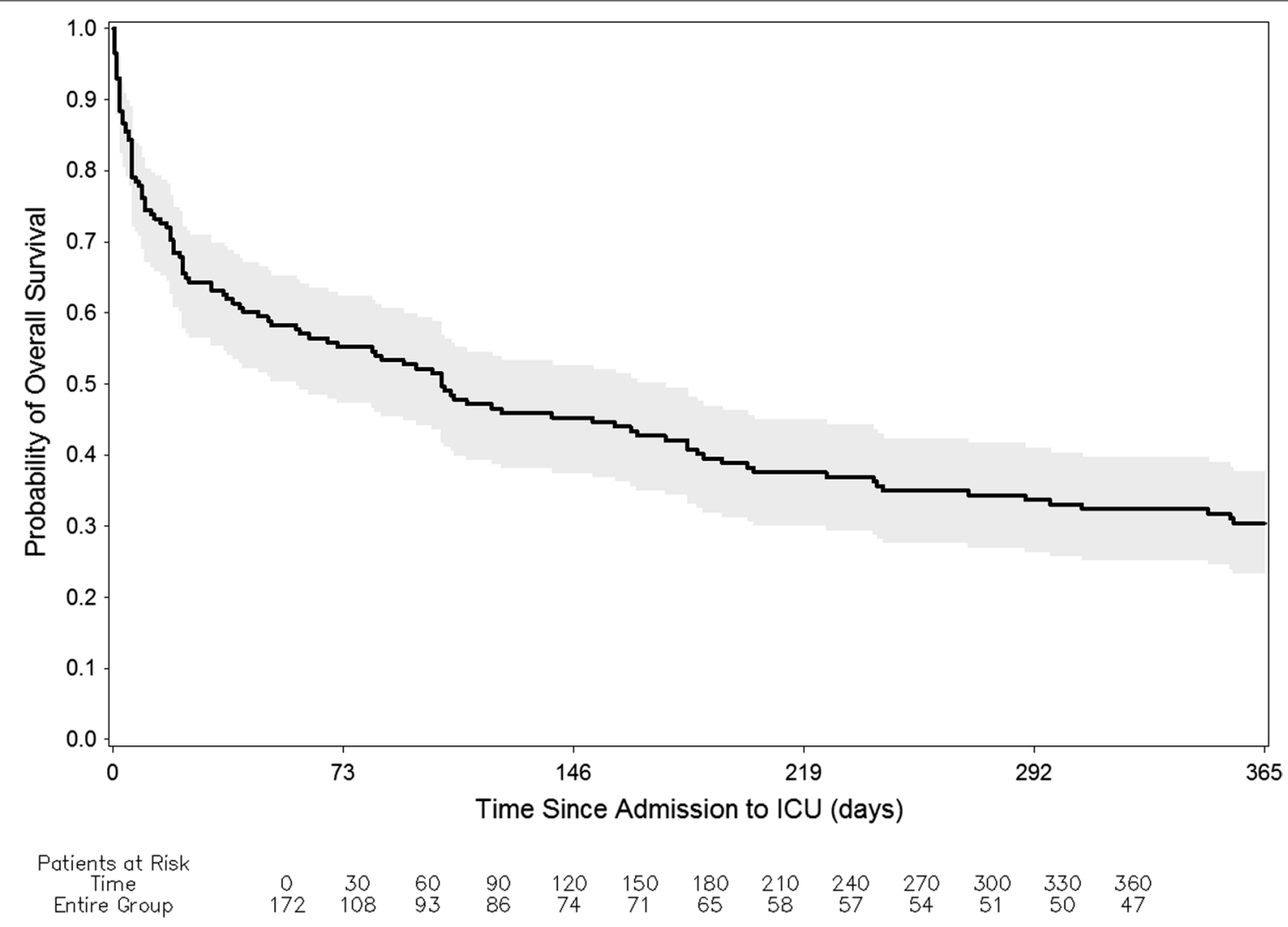

Median survival=104 days ( $95 \% \mathrm{Cl} 58-175$ days)

Fig. 1 Kaplan-Meier estimates of overall survival 
Table 5 Multivariable cox proportional hazards model for overall survival

\begin{tabular}{|c|c|c|c|c|}
\hline \multirow{2}{*}{$\begin{array}{l}\text { Variable } \\
\text { Histological diagnosis }\end{array}$} & \multirow[t]{2}{*}{$\begin{array}{l}\text { Hazard } \\
\text { ratio }\end{array}$} & \multicolumn{2}{|c|}{$\begin{array}{l}95 \% \mathrm{Cl} \\
\mathrm{P}\end{array}$} & p \\
\hline & & & & 0.006 \\
\hline $\begin{array}{l}\text { Unclassified high-grade } \\
\text { sarcoma }\end{array}$ & 1.000 & & & \\
\hline Bones $^{\mathrm{a}}$ & 0.881 & 0.516 & 1.506 & 0.64 \\
\hline Vascular ${ }^{b}$ & 1.657 & 0.862 & 3.185 & 0.13 \\
\hline GIST & 0.281 & 0.119 & 0.662 & 0.004 \\
\hline $\mathrm{MFH}$ & 0.674 & 0.236 & 1.929 & 0.46 \\
\hline Muscle & 2.048 & 0.917 & 4.573 & 0.08 \\
\hline Leiomyosarcoma & 0.375 & 0.160 & 0.880 & 0.02 \\
\hline Liposarcoma & 1.412 & 0.575 & 3.468 & 0.45 \\
\hline Synovial sarcoma & 0.793 & 0.293 & 2.143 & 0.65 \\
\hline Others & 0.746 & 0.373 & 1.493 & 0.41 \\
\hline Status of malignancy & & & & 0.003 \\
\hline Complete remission & 1.000 & & & \\
\hline First course of chemotherapy & 2.323 & 0.473 & 11.425 & 0.30 \\
\hline Mixed response & 2.720 & 0.444 & 16.677 & 0.28 \\
\hline Progression & 5.172 & 1.075 & 24.874 & 0.04 \\
\hline Stable disease & 2.262 & 0.501 & 10.218 & 0.29 \\
\hline \multicolumn{5}{|c|}{ Reason for ICU admission pancytopenia } \\
\hline \multicolumn{5}{|l|}{ No } \\
\hline Yes & 0.351 & 0.226 & 0.546 & $<0.0001$ \\
\hline \multicolumn{5}{|c|}{ Reason for ICU admission respiratory failure } \\
\hline \multicolumn{5}{|l|}{ No } \\
\hline Yes & 1.703 & 1.125 & 2.579 & 01 \\
\hline $\mathrm{CCl}$ (continuous) & 1.139 & 1.023 & 1.268 & 02 \\
\hline $\begin{array}{l}\text { SOFA score at discharge (con- } \\
\text { tinuous) }\end{array}$ & 1.210 & 1.141 & 1.283 & $<0.0001$ \\
\hline
\end{tabular}

Initially included in the model and then reduced by stepwise selection method: histology diagnosis, status of malignancy, number of organ metastasis, number of organ failures, and reason for ICU admission: acidosis, acute renal failure, pancytopenia, respiratory failure, and septic shock. There were 13 patients without known malignancy status who were not included in this analysis

Cl confidence interval, GIST Gastrointestinal stromal tumor, MFH Malignant fibrous histiocytoma, CCI Charlson comorbidities index, SOFA Sequential Organ Failure Assessment

a Ewing sarcoma, osteosarcoma, chondrosarcoma

${ }^{b}$ Angiosarcoma, epithelioid hemangioendothelioma

sarcoma. There appears to be significant heterogeneity in ICU mortality among all cancer patients because of a lack of data comparing similar histologies. Our patient population had an ICU mortality rate of $23 \%$, which is comparable to the $20 \%$ mortality in solid tumors [10]. The same study reported that ICU and in-hospital mortality rates for cancer patients were not significantly different from those of non-cancer patients [10]. However, among patients with multiple organ failures; mortality rates were higher in cancer patients than in non-cancer patients. This finding is consistent with our study, in which the number of organ failures was directly proportional to
ICU mortality. Among mortality statistics reported for specific tumors, patients with head and neck cancer, lung cancer, and gynecological malignancies have been shown to have ICU mortality rates of 39,36 , and $17.3 \%$, respectively [11-13]. In contrast, patients with hematological cancers have been reported to have higher ICU mortality rates: 48.3 and $56 \%$ in two separate studies [14, 15]. These higher rates may be attributed to increased severity of illness and higher incidence of sepsis due to associated leukopenia in these patients [10].

Several ICU scores; including MODS, APACHE II, SAPS II, and SOFA, have been used as objective ways to describe mild to severe organ dysfunction [16-19]. In our study, we used SOFA scores, which have been calibrated to predict ICU and in-hospital mortality rates in cancer patients by evaluating the combination of clinical conditions that lead to ICU admission [20-23].

We found out those patients who had high SOFA scores on admission did worse than patients with lower SOFA scores. Another important objective finding was that patients whose SOFA scores continued to rise following admission to the ICU had the worst outcomes, making these patients appropriate candidates for early supportive and palliative care. As a general guide, increase in scores of 6 or more since admission led to drastic changes in outcome and ICU survival decreased from 60 to $13.3 \%$ (Table 3). A patient who is admitted with a SOFA score of 11 or higher and their score increases by 3 or more has dismal ICU survival of $12.5 \%$. Such a patient should be considered for transition to hospice (Table 3).

Among individual clinical findings on ICU admissions, patients with cardiac arrest had the worst prognosis, with an ICU mortality rate of $72 \%(8 / 11)$ and a median OS of $24 \mathrm{~h}$. However, cardiopulmonary resuscitation has been shown to be a non-beneficial intervention in more than $90 \%$ of the patients with cancer [24-26]. In our univariate analysis, patients with respiratory failure had worse outcomes than patients with good respiratory status. Mechanical ventilation has been shown to be associated with increased mortality rates (73\%) in cancer patients, especially those with disseminated disease and poor performance status at the time of ICU admission [27]. Similar findings have been observed in patients with hematological, lung, and head and neck cancers in the ICU setting (64-74\%) [20, 28-30].

Hypotension and septic shock are additional clinical findings that correlated with higher ICU mortality in our multivariate analysis. Sepsis is one of the leading causes of ICU admission in cancer patients. However, patients with infections leading to shock and vasopressor use tend to do poorly and should be managed conservatively [10, 13, 15, 27]. Patients with acute kidney injury also have high acute mortality. This trend is more commonly seen 
in patients with hematological malignancies [31]. Furthermore, renal replacement therapy has been associated with high ICU mortality [24]. Given their bleak prognoses, patients with these individual or combinations of clinical findings should be triaged early so that inappropriate use of aggressive therapy can be avoided.

There are limited data evaluating long-term survival rates of critically ill cancer patients after their discharge from the ICU. Six-month OS in sarcoma patients was $41 \%$ in our study, which is comparable to the 6-month mortality rates of 59.3 and $66 \%$ reported in critically ill patients with hematological malignancies $[28,30]$. However, long-term mortality rates of 63 to $98 \%$ have been reported in patients with lung cancer. The major predictors of long-term prognosis in these patients were dependence on mechanical ventilation during the ICU stay and progression of cancer after discharge from the ICU [11]. In our study, we found that the status of malignancy, metastatic disease, Charlson comorbidity index and SOFA scores at discharge, and the presence of respiratory failure or cytopenia at the time of ICU admission were significant predictors of survival outcomes in both univariate and multivariate analyses (Table 4). Therefore, when looking at patterns of long-term survival after ICU stay, the above-mentioned clinical prognostic factors should be considered early in the clinical course.

There were a number of limitations to this study. First, this is a single-institution retrospective study covering a limited number of years. We are a tertiary care referral center for sarcomas, so the results of our study may not be applicable to smaller hospitals or low-volume centers. Each hospital has its own ICU admission and discharge policies which may bias the results of a single center study. Second, our study did not have a control group to compare the outcomes of critically ill sarcoma patients who were not admitted to the ICU and were managed conservatively. Even though it is a large collection of a rare tumor our study outcomes were limited to only 40 events in the ICU. Due to a small number of events and multiple risk factors, it is difficult to make a generalized presumption of which risk factor individually impacted the short term survival. In addition, quality-of-life measures and performance status scores could not be reliably collected in the retrospective setting.

In conclusion, our study is the largest study to date of OS in sarcoma patients admitted to the ICU. In our study span of 7 years, we have shown that OS in sarcoma is comparable with that of critically ill patients with other solid tumors. The admission SOFA scores and change in the SOFA scores during ICU stay are highly reliable indicators of probability of survival and should be used in decision making in critically ill patients. Our results suggest that patients with advanced malignancy that are admitted to the ICU for respiratory failure, cardiac arrest, septic shock, acute renal failure or acidosis and have high SOFA score with subsequent worsening in the ICU have very poor prognosis. Based on the retrospective data which needs further validation we can recommend that judicious approach should be taken in patients with predictors of poor survival before subjecting them to aggressive treatment.

\section{Authors' contributions}

$R G, D A, R B, S P, J L$, and VR were responsible for the study concept. All authors contributed to the study design. RG, NR, and EJR collected and assembled these data. NR, JS, and XL analyzed and interpreted these data. RG and NH wrote the initial draft, and all the authors approved the final draft of the report. All authors read and approved the final manuscript.

\section{Author details}

${ }^{1}$ The University of Texas at Houston Internal Medicine Residency Program, Houston, TX, USA. ${ }^{2}$ The University of Texas Graduate School of Biomedical Sciences at Houston, Houston, TX, USA. ${ }^{3}$ Division of Quantitative Sciences, The University of Texas MD Anderson Cancer Center, Houston, TX, USA. ${ }^{4}$ Department of Critical Care, The University of Texas MD Anderson Cancer Center, Houston, TX, USA. ${ }^{5}$ Department of Sarcoma Medical Oncology, The University of Texas MD Anderson Cancer Center, 1515 Holcombe Blvd \# 450, Houston, TX 77030, USA.

\section{Acknowledgements}

We did not have any financial support. We thank critical care team for providing the initial data and SOFA scores. We thank statisticians Naveen Ramesh, Xiudong Lei and Juhee Song. The statistical analysis work was supported in part by the Cancer Center Support Grant (NCI Grant P30 CA016672). We thank scientific editors, Bryan Tutt and Tamara Locke, in Department of Scientific

Publications at MD Anderson for manuscript editing.

Poster presented at 2014 American Society of Clinical Oncology National Meeting, Chicago, IL.

\section{Competing interests}

The authors declare that they have no competing interests.

Received: 5 March 2016 Accepted: 17 June 2016

Published online: 19 July 2016

\section{References}

1. Fletcher CDM, Bridge JA, Hogendoorn P, Mertens F. World Health Organization classification of tumors of soft tissue and bone. 4th ed. Lyon: IARC Press; 2013.

2. Mackall CL, Meltzer PS, Helman LJ. Focus on sarcomas. Cancer Cell. 2002;2:175-8

3. Chassin MR. Costs and outcomes of medical intensive care. Med Care. 1982;20:165-79.

4. Turnbull A, Goldiner P, Silverman D, et al. The role of an intensive care unit in a cancer center. An analysis of 1035 critically ill patients treated for lifethreatening complications editorial. Cancer. 1976;37:82-4.

5. Shapira DV, Studnicki J, Bradham DD. Intensive care, survival and expense of treating critically ill cancer patients. JAMA. 1993;269:783-6.

6. Azoulay E, Moreau D, Alberti C, et al. Predictors of short-term mortality in critically ill patients with solid malignancies. Intensive Care Med. 2000;26:1817-23.

7. Lecuyer $L$, Chevret S, Thiery G, et al. The ICU trial: a new admission policy for cancer patients requiring mechanical ventilation. Critical Care Med. 2007;35:808-14.

8. Azoulay E, Soares M, Darmon M, et al. Intensive care of the cancer patient: recent achievements and remaining challenges. Ann Intensive Care. 2011;1:5. 
9. Charlson ME, Pompei P, Ales KL, et al. A new method of classifying prognostic comorbidity in longitudinal studies: development and validation. J Chronic Dis. 1987;40:373-83.

10. Taccone FS, Artigas AA, Sprung CL, et al. Caracteristics and outcomes of cancer patients in European ICUs. Crit Care. 2009;13:R15.

11. Soubani AO, Ruckdeschel JC. The outcome of medical intensive care for lung cancer patients: the case for optimism. J Thorac Oncol. 2011;6:633-8.

12. Namendys-Silva SA, González-Herrera MO, Texcocano-Becerra J, et al. Outcomes of critically ill gynecological cancer patients admitted to intensive care unit. Am J Hosp Palliat Care. 2013;30:7-11.

13. Soares M, Salluh JI, Ferreira CG, et al. Prognosis of lung cancer patients with life-threatening complications. Chest. 2007;131:840-6.

14. Pène $F$, Azoulay $E$, Blot $F$, et al. Outcome of critically ill allogeneic hematopoietic stem-cell transplantation recipients: a reappraisal of indications for organ failure supports. J Clin Oncol. 2006;24:643-9.

15. Geerse DA, Span LF, Pinto-Sietsma SJ, et al. Prognosis of patients with haematological malignancies admitted to the intensive care unit: sequential Organ Failure Assessment (SOFA) trend is a powerful predictor of mortality. Eur J Intern Med. 2011;22:57-61.

16. Marshall JC, Cook DJ, Christou NV, et al. Multiple organ dysfunction score: a reliable descriptor of a complex clinical outcome. Crit Care Med. 1995;23:1638-52.

17. Vincent JL, Moreno R, Takala J, et al. The SOFA (Sepsis-related Organ Failure Assessment) score to describe organ dysfunction/failure. On behalf of the Working Group on Sepsis-Related Problems of the European Society of Intensive Care Medicine. Intensive Care Med. 1996;22:707-10.

18. Knaus WA, Draper EA, Wagner DP, et al. APACHE II: a severity of disease classification system. Crit Care Med. 1985;13:818-29.

19. Le Gall JR, Lemeshow S, Saulnier F. A new simplified acute physiology score (SAPS II) based on a European/North American multicenter study. JAMA. 1993;270:2957-63.

20. Reichner CA, Thompson JA, O'Brien S, et al. Outcome and code status of lung cancer patients admitted to the medical ICU. Chest. 2006;130:719-23.

21. Soares M, Caruso P, Silva E, et al. Characteristics and outcomes of patients with cancer requiring admission to intensive care units: a prospective multicenter study. Crit Care Med. 2010;38:9-15.
22. Owczuk R, Wujtewicz MA, Sawicka W, et al. Patients with haematological malignancies requiring invasive mechanical ventilation differences between survivors and non-survivors in intensive care unit. Support Care Cancer. 2005;13:332-8.

23. Cárdenas-Turanzas M, Ensor J, Wakefield C, et al. Cross-validation of a sequential organ failure assessment score-based model to predict mortality in patients with cancer admitted to the intensive care unit. J Crit Care. 2012;27:673-80.

24. Heo SJ, Kim G, Lee CK, et al. Prediction of short- and long-term survival for advanced cancer patients after ICU admission. Support Care Cancer. 2014;1:1-9.

25. Wallace S, Ewer MS, Price KJ, et al. Outcome and cost implications of cardiopulmonary resuscitation in the medical intensive care unit of a comprehensive cancer center. Support Care Cancer. 2002;10:425-9.

26. Reisfield GM, Wallace SK, Munsell MF, et al. Survival in cancer patients undergoing in-hospital cardiopulmonary resuscitation: a meta-analysis. Resuscitation. 2006;71:152-60.

27. Azevedo LC, Caruso P, Silva UV, et al. Outcomes for patients with cancer admitted to the ICU requiring ventilatory support: results from a prospective multicenter study. Chest. 2014;146:257-66.

28. Bird GT, Farquhar-Smith P, Wigmore T, et al. Outcomes and prognostic factors in patients with hematological malignancy admitted to a specialist cancer intensive care unit: a 5 yr study. Br J Anesth. 2012;108:452-9.

29. Soares M, Salluh Jl, Toscano L, et al. Outcomes and prognostic factors in patients with head and neck cancer and severe acute illnesses. Intensive Care Med. 2007;33:2009-13.

30. Benoit DD, Vandewoude KH, Decruyenaere JM, et al. Outcome and early prognostic indicators in patients with a hematologic malignancy admitted to the intensive care unit for a life-threatening complication. Crit Care Med. 2003;31:104-12

31. Hampshire PA, Welch CA, McCrossan LA, et al. Admission factors associated with hospital mortality in patients with haematological malignancy admitted to UK adult, general critical care units: a secondary analysis of the ICNARC Case Mix Programme Database. Crit Care. 2009;13:1-17.

\section{Submit your next manuscript to BioMed Central and we will help you at every step:}

- We accept pre-submission inquiries

- Our selector tool helps you to find the most relevant journal

- We provide round the clock customer support

- Convenient online submission

- Thorough peer review

- Inclusion in PubMed and all major indexing services

- Maximum visibility for your research

Submit your manuscript at www.biomedcentral.com/submit
(O) BioMed Central 